\title{
HAUSDORFF DIMENSION OF BANACH SPACES
}

\author{
by J. ARIAS DE REYNA
}

(Received 29th July 1986)

\section{Introduction}

We show that if $X$ is a Banach space of infinite dimension and $\mu^{h}$ is a Hausdorff measure, where $h$ is continuous, then there exists a measurable set $K \subset X$ such that $0<\mu^{h}(K)<+\infty$. We also characterize the normed spaces in which the unit ball can be covered by a sequence of balls whose radii $r_{n}<1$ converge to zero as $n \rightarrow \infty$.

First we define the concept of a Hausdorff small metric space. We say that a metric space $(X, d)$ is Hausdorff small if there exists a sequence of balls of $X,\left(B\left(x_{n}, r_{n}\right)\right)$, such that $\lim r_{n}=0$ and $X=\bigcup_{n \geqq N} B\left(x_{n}, r_{n}\right)$ for every natural number $N$. We prove that a metric space $X$ is Hausdorff small if, and only if, there exists $h \in \mathscr{H}_{0}$ such that the Hausdorff measure $\mu^{h}$ satisfies $\mu^{h}(X)=0$. (Here $\mathscr{H}_{0}$ is the class of monotonic increasing functions $h:[0,+\infty) \rightarrow[0,+\infty]$, continuous on the right such that $h(0)=0$ and $h(t)>0$ for every $t>0$ (Rogers [8]).) For brevity we will say small metric space instead of Hausdorff small metric space.

We see then that the family of small subspaces of $X$ is a $\sigma$-ideal and prove that the $\sigma$ ideal of small subspaces is larger than that generated by precompact subspaces.

The $\sigma$-ideal of the small subsets of a metric space $X$ has many properties in common with those of the sets of first category or those of zero measure. For instance, in a separable metric space, assuming Martin's hypothesis, the union of fewer than continuum many small sets is small. We want to thank A. Durán Guardeño for permitting us to include his proof of this fact.

The main theorem of this paper is that if $G$ is a non-locally compact complete metric group with an invariant metric $d$ and $h \in \mathscr{H}_{0}$ is a continuous function, there exists a compact subset $K \subset X$ such that $0<\mu^{h}(K)<+\infty$. As a consequence, the unit ball of a Fréchet space $X$ is small if, and only if, $X$ is finite dimensional.

It also follows that, in a non-locally compact Polish group, a universally measurable small subset is a Haar zero set in the sense of Christensen [1]. We prove also that there exists a Haar zero set which is not small.

We further prove that a dense hyperplane of a Banach space is not small. The proof of this fact depends on results of Banach space theory.

Finally it is proved that the unit ball of a normed space $X$ can be covered by a sequence of balls whose radii $r_{n}<1$ converge to zero with $n$ if, and only if, $X$ is small. We must observe that T. W. Körner [7] previously proved that a Banach space of infinite dimension cannot be covered by a sequence of balls whose radii $r_{n}<1$ converge to zero. Later J. Connett $[2,3]$ obtained some related results, in particular he proved that the unit ball of a Banach space of infinite dimension cannot be covered by a collection of subsets $\left\{D_{i}\right\}_{i=1}^{\infty}$ of diameter $\operatorname{diam}\left(D_{i}\right)<2$ such that $\lim \operatorname{diam}\left(D_{i}\right)=0$. 


\section{Small metric spaces}

Definition 1. Let $(X, d)$ be a metric space. We say that $X$ is small if there exists a sequence of balls $B\left(x_{n}, r_{n}\right)$ such that:

(1) $\lim r_{n}=0$.

(2) For every $N, \bigcup_{n \geqq N} B\left(x_{n}, r_{n}\right)=X$.

If $(X, d)$ is small, $\left\{x_{n}: n \in \mathbb{N}\right\}$ is countable and dense. So $(X, d)$ is a separable space.

Proposition 2. If $(X, d)$ is totally bounded, it is small.

Proof. For every $n$, there exists a finite number of balls $B\left(x_{k}^{n}, 1 / n\right)$ that cover $X$ : $X=\bigcup_{k=1}^{K_{n}} B\left(x_{k}^{n}, 1 / n\right)$. Now it is enough to consider the sequence

$$
B\left(x_{1}^{1}, 1\right), \ldots, B\left(x_{K_{1}}^{1}, 1\right), B\left(x_{1}^{2}, 1 / 2\right), \ldots, B\left(x_{K_{2}}^{2}, 1 / 2\right), B\left(x_{1}^{3}, 1 / 3\right), \ldots
$$

If $(X, d)$ is a metric space, we say that $A \subset X$ is small if the space $A$ with the induced metric is small.

Proposition 3. Let $(X, d)$ be a metric space and $A \subset X . A$ is small if, and only if, there exists a sequence of balls of $X\left(B\left(x_{n}, r_{n}\right)\right)$ such that:

(1) $\lim r_{n}=0$.

(2) For every $N, A \subset \bigcup_{n \geqq N} B\left(x_{n}, r_{n}\right)$.

Proof. If $A$ is small, there exist balls with centres in $A$ satisfying (1) and (2).

If there exist balls of $X$ that satisfy (1) and (2), for every $n$ such that $A \cap B\left(x_{n}, r_{n}\right) \neq \emptyset$, we choose $y_{n} \in A \cap B\left(x_{n}, r_{n}\right)$ and, for every $n$ with $A \cap B\left(x_{n}, r_{n}\right)=\emptyset$, we choose $y_{n} \in A$ arbitrarily. We consider the balls of $A B_{A}\left(y_{n}, 2 r_{n}\right)$. We have $\lim 2 r_{n}=0$ and also, for every $N, A=\bigcup_{n \geqq N} B_{A}\left(x_{n}, r_{n}\right) \subset \bigcup_{n \geqq N} B_{A}\left(y_{n}, 2 r_{n}\right) \subset A$.

Proposition 4. Let $(X, d)$ be a metric space. The collection $\mathcal{N}=\{A \subset X: A$ is small $\}$ is a $\sigma$-ideal.

Proof. It is obvious that $\emptyset \in \mathscr{N}$. Let $B \subset A \in \mathscr{N}$. By Proposition 3 we have $B \in \mathscr{N}$.

Let $A_{n} \in \mathscr{N}$ for every $n \in \mathbb{N}$. Then there exist balls $B\left(x_{k}^{n}, r_{k}^{n}\right)$ such that $\lim r_{k}^{n}=0$ and $A_{n} \subset \bigcup_{k \geqq K} B\left(x_{k}^{n}, r_{k}^{n}\right)$ for every $K \in \mathbb{N}$. We can suppose that, for every $n, r_{k}^{n}<1 / n$. If we arrange the balls $B\left(x_{k}^{n}, r_{k}^{n}\right)$ in a sequence $\left(B\left(z_{m}, \rho_{m}\right)\right)$ we have:

(1) $\lim \rho_{m}=0$. Indeed for every $\varepsilon \in 0$, there exists $N$ such that $1 / N<\varepsilon$. So $r_{k}^{n}<\varepsilon$ for every $n \geqq N$ and $k \in \mathbb{N}$. Further, for every $i \in\{1,2, \ldots, N-1\}, \lim _{k \rightarrow \infty} r_{k}^{i}=0$. Thus $\rho_{m}>\varepsilon$ only for finitely many indices $m$.

(2) For every $M>0$ and every $n$, there exists $K(n)$ such that $\bigcup_{m \geqq M} B\left(z_{m}, \rho_{m}\right)>$ $\bigcup_{k \geqq K(n)} B\left(x_{k}^{n}, r_{k}^{n}\right)$ and it follows that $\bigcup_{m \geqq M} B\left(z_{m}, \rho_{m}\right) \supset \cup A_{n}$.

Thus $\cup A_{n}$ is a small metric space. 
Theorem 5. (Assuming Martin's hypothesis). For $\alpha<\kappa$ let $A_{\alpha}$ be small subspaces of a separable metric space $(X, d)$. Then $\cup A_{\alpha}$ is small if $\kappa<2^{\omega}$.

Proof. (A. Durán Guardeño). Let $\left(B\left(z_{n}, \delta_{n}\right)\right)$ be a sequence which consists of all the balls with centres in a fixed countable dense subset of $X$ and rational radii.

Since $A_{\alpha}$ is small, $A_{\alpha} \subset \bigcap_{N} \bigcup_{n \geqq N} B\left(x_{n}^{\alpha}, r_{n}^{\alpha}\right)$ and $\lim _{n \rightarrow \infty} r_{n}^{\alpha}=0$. We can assume without loss of generality that, for every $\alpha$ and $n$, there exists $m \in \mathbb{N}$ such that $x_{n}^{\alpha}=z_{m}$ and $r_{n}^{\alpha}=\delta_{m}$, so $B\left(X_{n}^{\alpha}, r_{n}^{\alpha}\right)=B\left(z_{m}, \delta_{m}\right)$.

We define the sets $a_{m}=\left\{n \in \omega: \delta_{n}<1(m+1)\right\}, c_{\alpha}=\{n \in \omega$ : there exists $k$ such that $\left.B\left(x_{k}^{\alpha}, r_{k}^{\alpha}\right)=B\left(z_{n}, \delta_{n}\right)\right\}$ and the subsets $\mathscr{A}=\left\{a_{m}: m \in \omega\right\}, \mathscr{B}=\mathscr{D}=\{\omega\}$ and $\mathscr{C}=\left\{c_{\alpha}: \alpha<\kappa\right\}$ of $\mathscr{P}(\omega)$.

Now we use a proposition which is valid assuming Martin's hypothesis.

Let $\mathscr{A}, \mathscr{B}, \mathscr{C}, \mathscr{D}$ be families of subsets of $\omega$ such that

(1) $A \cap C$ is finite for every $A \in \mathscr{A}$ and $C \in \mathscr{C}$;

(2) $B \backslash \cup \mathscr{A}_{0}$ is infinite for every $B \in \mathscr{B}$ and every finite family $\mathscr{A}_{0} \subset \mathscr{A} \backslash\{B\}$;

(3) $D \backslash \cup \mathscr{C}_{0}$ is infinite for every $D \in \mathscr{D}$ and every finite family $\mathscr{C}_{0} \subset \mathscr{C} \backslash\{D\}$;

(4) $\mathscr{A}$ is countable and $\mathscr{B}, \mathscr{C}$ and $\mathscr{D}$ have cardinals less than the cardinal of the continuum.

Then there is an $I \subset \omega$ such that:

$A \backslash I$ is finite if $A \in \mathscr{A}$;

$B \backslash I$ is non-empty if $B \in \mathscr{B}$, infinite if $B \in \mathscr{B} \backslash \mathscr{A}$;

$C \cap I$ is finite if $C \in \mathscr{C}$;

$D \cap I$ is non-empty if $D \in \mathscr{D}$, infinite if $D \in \mathscr{D} \backslash \mathscr{C}$.

(Fremlin [5, p. 30].)

We check that the hypotheses of this theorem are verified.

(1) $a_{m} \cap c_{\alpha}$ is a finite set for every $m \in \omega$ and $\alpha<\kappa$. Since $\lim _{n \rightarrow \infty} r_{n}^{\alpha}=0, r_{n}^{\alpha}>1 / m$ only for a finite number of indices $n$.

(2) $\left|\omega \backslash \bigcup_{m \in F} a_{m}\right|=\omega$ for every finite subset $F \subset \omega$. This follows easily because $\bigcup_{m \in F} a_{m}=$ $a_{p}$ if $p=\max F$.

(3) $\left|\omega \backslash \bigcup_{\alpha \in F} c_{\alpha}\right|=\omega$ for every finite subset $F \subset \kappa$, since every $c_{\alpha}$ contains only a finite number of indices $n$ such that $\delta_{n}>1$.

(4) By definition, $\mathscr{A}$ is countable and $\mathscr{B}, \mathscr{C}$ and $\mathscr{D}$ have cardinal numbers less than that of the continuum.

Let $I$ be the set obtained in the theorem and let $P=\omega \backslash I$.

In order to prove that $\cup A_{\alpha}$ is small we are going to prove the following assertions:

(a) $\lim _{\substack{n \rightarrow \infty \\ n \in P}} \delta_{n}=0$.

Indeed, given $m \in \mathbb{N}$, since $a_{m} \cap P$ is finite it follows that $\delta_{n} \leqq 1 /(m+1)$ for every $n \in P$ except for a finite number of indices. 
(b) $\bigcup_{\alpha<\kappa} A_{\alpha} \subset \bigcap_{N} \bigcup_{\substack{n \geq N \\ n \in P}} B\left(z_{n}, \delta_{n}\right)$.

Let $P_{N}=\{n \in P: n \geqq N\}$ for every $N$ and let $\alpha<\kappa$. Since $c_{\alpha} \backslash P$ is a finite set, the set of balls $\left\{B\left(z_{n}, \delta_{n}\right): n \in P_{N}\right\}$ contains the set of balls $\left\{B\left(x_{n}, r_{n}\right): n \in \omega\right\}$ except for a finite number. So $A_{\alpha} \subset \bigcap_{N} \bigcup_{n \in P_{N}} B\left(z_{n}, \delta_{n}\right)$.

Thus $\cup A_{\alpha}$ is small.

\section{Small metric spaces and Hausdorff measures}

Let $\mathscr{H}$ denote the class of functions from $[0,+\infty) \rightarrow[0,+\infty]$, monotonic increasing for $t \geqq 0$, positive for $t>0$ and continuous on the right for all $t \geqq 0$. Let $\mathscr{H}_{0}$ be the subset of all $h \in \mathscr{H}$ such that $h(0)=0$. Every $h \in \mathscr{H}$ defines a metric exterior measure $\mu^{h}$, called a Hausdorff measure (Rogers [6]).

Proposition 6. Let $(X, d)$ be a metric space. Then $X$ is small if, and only if, there exists $h \in \mathscr{H}_{0}$ (continuous) such that $\mu^{h}(X)=0$.

Proof. Let $h \in \mathscr{H}_{0}$ be such that $\mu^{h}(X)=0$. Then there exists a sequence $\left(E_{n}\right)$ of sets with $\sum h\left(\operatorname{diam}\left(E_{n}\right)\right)<+\infty$ so that each point of $X$ belongs to infinitely many of the sets $E_{n}$ (Rogers [6, p. 59]).

Let $x_{n} \in E_{n}$ and $r_{n}=\operatorname{diam}\left(E_{n}\right)$. We have $X=\bigcap_{N} \bigcup_{n \geqq N} B\left(x_{n}, r_{n}\right)$ and $\lim r_{n}=0$. So $X$ is small.

If $X$ is small there exists a sequence $\left(B\left(x_{n}, r_{n}\right)\right)$ of balls such that $X=\bigcap_{N} \bigcup_{n \geqq N} B\left(x_{n}, r_{n}\right)$ and $\lim r_{n}=0$. We can assume $r_{n}$ decreasing. Since $\lim r_{n}=0$, there exists $h \in \mathscr{H}_{0}$ such that $h\left(2 r_{n}\right)=2^{-n}$. We can assume also that $h$ is continuous. Now $X=\bigcap_{N} \bigcup_{n \geqq N} B\left(x_{n}, r_{n}\right)$ and $\sum h\left(\operatorname{diam}\left(B\left(x_{n}, r_{n}\right)\right)\right)=\sum h\left(2 r_{n}\right)<+\infty$. So $\mu^{h}(X)=0$.

We now prove the main theorem of this paper. We shall need the concept introduced by Kuratowski [7] of $\alpha$-measure of a bounded subset of a metric space.

If $(X, d)$ is a metric space and $A \subset X$ is bounded, we define the $\alpha$-measure $\alpha(A)$ of $A$ as the infimum of the set of real numbers $\varepsilon>0$ such that $A$ can be covered by a finite number of sets of diameter less than $\varepsilon$.

An infinite subset $A$ of $X$ is said to be $\alpha$-minimal if $\alpha(A)=\alpha(B)$ for every infinite subset $B$ of $A$. This concept is due to Dominguez Benavides [4]. In [4] it is proved that, if $X$ is an infinite and bounded metric space, then there exists an $\alpha$-minimal subset $A$ of $X$. Furthermore, if $X$ is not totally bounded there exists an $\alpha$-minimal set $A$ such that $\alpha(A)>0$. Finally, if $A$ is an $\alpha$-minimal subset, then for every positive number $\varepsilon$, there exists an infinite subset $B$ of $A$ such that $\alpha(A)-\varepsilon<d(x, y)<\alpha(A)+\varepsilon$ for every $x \in B, y \in B$, $x \neq y$.

Theorem 7. Let $(G, d)$ be a non-locally compact complete metric group whose metric d is left invariant. Let $h$ be a continuous function in $\mathscr{H}_{0}$. There exists a compact subset $L \subset G$ such that $0<\mu^{h}(L)<+\infty$, where $\mu^{h}$ is the Hausdorff measure.

Proof. Since $G$ is not locally compact, every ball contains an $\alpha$-minimal subset $M$ such that $\alpha(M)>0$. So there exists a sequence $\left(M_{n}\right)$ of $\alpha$-minimal subsets satisfying 
$\alpha\left(M_{n}\right)>0$ for every $n, \alpha\left(M_{n}\right)$ converges to zero and $e \in M_{n} \subset B(e, 1 / n)$, where $e$ is the unit element of $G$.

We denote by $S$ the set of finite sequences of $\omega=\{0,1,2, \ldots\}$. The sequence of 0 elements coincides with the empty set and belongs to $S$. If $s \in S$, we denote by $|s|$ the length of the sequence $s$. If $s \in S$ and $n \in \omega$ we denote by $s n$ the sequence of length $|s|+1$ that extends $s$ and whose last term is $n$. If $s, t \in S$ we denote by st the sequence of length $|s|+|t|$ and whose terms are those of $s$ followed by those of $t$.

Choose $\varepsilon>0$ such that $6 \varepsilon<h\left(\alpha\left(M_{2}\right)\right)$ and, for every $s \in S$, choose $\varepsilon_{s}>0$ such that $\varepsilon=$ $\sum_{s \in S} \varepsilon_{s}$

We will define; by induction on the length of $s$, for every $s \in S$, a ball $B\left(x_{s}, r_{s}\right)$, a real number $\delta_{s}>0$, and a natural number $J(s)$ such that, if $\alpha_{s}=\alpha\left(M_{J(s)}\right)$, we have:

(1) $x_{\theta}=e, r_{\theta}=1$;

(2) $\left|t-\alpha_{s}\right|<\delta_{s}$ implies $\left|h(t)-h\left(\alpha_{s}\right)\right|<\varepsilon_{s}$;

(3) $0<\delta_{s}<\alpha_{s}, 2 \delta_{s}<3 r_{s}$ and $r_{s n}=\delta_{s} / 3$;

(4) $\alpha_{s}-\delta_{s} / 3<d\left(x_{s n}, x_{s m}\right)<\alpha_{s}+\delta_{s} / 3$ if $n \neq m$, and $d\left(x_{s n}, x_{s}\right)<r_{s} / 2$;

(5) $h\left(\alpha_{s}\right)=\sum_{n=0}^{\infty} h\left(\alpha_{s n}\right)$;

(6) $\left\{x_{s n}: n \in \omega\right\}$ is an $\alpha$-minimal set of $\alpha$-measure $\alpha_{s}$.

To begin the induction, condition (1) determines the centre and radius of $B\left(x_{\emptyset}, r_{\emptyset}\right)$, we now choose $J(\emptyset)=2$ and $\alpha_{\emptyset}=\alpha\left(M_{2}\right)$. It is clear that $M_{2} \subset B(e, 1 / 2)=B\left(x_{\emptyset}, r_{\emptyset} / 2\right)$. Finally $\delta_{\emptyset}$ is chosen using the continuity of $h$ so that (2) and (3) are satisfied.

Suppose we have already defined the centre and radius of the ball $B\left(x_{s}, r_{s}\right)$, and we know $J(s), \alpha_{s}$ and $\delta_{s}$, we now define $x_{s n}, r_{s n}, \alpha_{s n}, \delta_{s n}$ and $J(s n)$ for every $n$.

Since $\alpha\left(M_{n}\right)$ and $\operatorname{diam}\left(M_{n}\right)$ converge to zero, we can choose $J(s n)$ such that $\operatorname{diam}\left(M_{J(s n)}\right)<\delta_{s} / 6$ and also, if $\alpha_{s k}=\alpha\left(M_{J(s n)}\right)$, then $h\left(\alpha_{s}\right)=\sum_{n \in \omega} h\left(\alpha_{s n}\right)$. To see that this can be done we use that $h(t)>0$ for every $t>0, h(0)=0, h$ is continuous and that we can choose $\alpha_{s n}=\alpha_{s m}$ although $n \neq m$. We let $r_{s n}=\delta_{s} / 3$ and then $\operatorname{diam}\left(M_{J(s n)}\right)<r_{s n} / 2$.

Now we take a countably infinite subset $A \subset M_{J_{(s)}}$ such that $x, y \in A, x \neq y$ implies $\alpha_{s}-\delta_{s} / 3<d(x, y)<\alpha_{s}+\delta_{s} / 3$. We write $x_{s} A=\left\{x_{s n}: n \in \omega\right\}$. It is clear that, since $M_{J(s)} \subset B\left(e, r_{s} / 2\right),(4)$ and (6) hold. Here we are using the fact that $G$ is a group.

As we have already defined $\alpha_{s n}$, we can, because of the continuity of $h$, find $\delta_{s n}$ satisfying (2) and (3).

We observe that $B\left(x_{s}, r_{s}\right) \supset B\left(x_{s n}, r_{s n}\right)$. Indeed, if $x \in B\left(x_{s n}, r_{s n}\right)$ we have $d\left(x, x_{s}\right) \leqq d\left(x, x_{s n}\right)+$ $d\left(x_{s n}, x_{s}\right)<r_{s n}+r_{s} / 2=\delta_{s} / 3+r_{s} / 2<r_{s} / 2+r_{s} / 2=r_{s}$. Further, if $n \neq m, B\left(x_{s n}, r_{s n}\right) \cap B\left(x_{s m}, r_{s m}\right)=\emptyset$ because $d\left(x_{s n}, x_{s m}\right)>\alpha_{s}-\delta_{s} / 3>\delta_{s}-\delta_{s} / 3=\delta_{s} / 3+\delta_{s} / 3=r_{s n}+r_{s m}$. Finally the radii of these balls satisfy $r_{s n}=\delta_{s} / 3<r_{s} / 2$ and therefore $r_{s}<(1 / 2)^{|s|}$ and $r_{s}$ converges to zero as $|s| \rightarrow \infty$.

We define $L=\bigcap_{n=0} \bigcup_{|s|=n} B\left(x_{s}, r_{s}\right)$. This space is homeomorphic to $\mathcal{N}$, the space of all sequences of natural numbers, by the map $T: \mathcal{N} \rightarrow L$ which sends $t$ to the unique point of the set $\bigcap_{n=0}^{\infty} B\left(x_{t \mid n}, r_{t \mid n}\right)$. This set is a singleton because $r_{t \mid n}$ converges to zero as $n \rightarrow \infty$.

We now calculate the diameter of $L \cap B\left(x_{s}, r_{s}\right)$. If $x, y \in L \cap B\left(x_{s}, r_{s}\right), x \in B\left(x_{s n}, r_{s n}\right)$ and $y \in B\left(x_{s m}, r_{s m}\right)$ we have, supposing $n \neq m$,

$$
d(x, y) \leqq d\left(x, x_{s n}\right)+d\left(x_{s n}, x_{s m}\right)+d\left(x_{s m}, y\right)
$$




\section{J. ARIAS DE REYNA}

$$
\begin{aligned}
& \leqq r_{s n}+d\left(x_{s n}, x_{s m}\right)+r_{s m} \\
& \leqq \delta_{s} / 3+\alpha_{s}+\delta_{s} / 3+\delta_{s} / 3 \\
& =\alpha_{s}+\delta_{s},
\end{aligned}
$$

and

$$
\begin{aligned}
d(x, y) & \geqq d\left(x_{s n}, x_{s m}\right)-d\left(x, x_{s n}\right)-d\left(y, x_{s m}\right) \\
& \geqq \alpha_{s}-\delta_{s} / 3-2\left(\delta_{s} / 3\right) \\
& =\alpha_{s}-\delta_{s} .
\end{aligned}
$$

If $n=m$,

$$
d(x, y) \leqq 2 r_{s n}=2\left(\delta_{s} / 3\right)<2\left(\alpha_{s} / 3\right)<\alpha_{s}
$$

So

$$
\alpha_{s}-\delta_{s} \leqq \operatorname{diam}\left(\operatorname{Ln} B\left(x_{s}, r_{s}\right)\right) \leqq \alpha_{s}+\delta_{s}
$$

We write $B_{s}=B\left(x_{s}, r_{s}\right), L=\bigcup_{|s|=n} B_{s} \cap L$ and we have

$$
\left|\sum_{|s|=n} h\left(\operatorname{diam}\left(B_{s} \cap L\right)\right)-\sum_{|s|=n} h\left(\alpha_{s}\right)\right| \leqq \sum_{|s|=n} \varepsilon_{s}<\varepsilon .
$$

We also have

$$
\sum_{|s|=n} h\left(\alpha_{s}\right)=\sum_{|s|=n} \sum_{n=0}^{\infty} h\left(\alpha_{s k}\right)=\sum_{|t|=n+1} h\left(\alpha_{t}\right)=h\left(\alpha_{\theta}\right)
$$

and we obtain $\mu^{h}(L) \leqq h\left(\alpha_{0}\right)+\varepsilon<+\infty$.

We now prove that $\mu^{h}(L)>0$. We choose natural numbers $N(0), N(1), \ldots$ such that:

(a) $N(0)$ satisfies

$$
\left|h\left(\alpha_{0}\right)-\sum_{n=0}^{N(0)} h\left(\alpha_{n}\right)\right|<\varepsilon_{0} .
$$

(b) For every $k=0,1, \ldots, N(0)$, the number $N(1)$ satisfies

$$
\left|h\left(\alpha_{k}\right)-\sum_{n=0}^{N(1)} h\left(\alpha_{k n}\right)\right|<\varepsilon_{k} .
$$

(c) Assuming that we have chosen $N(0), N(1), \ldots, N(n-1)$, we choose $N(n)$ such that, 
for every $s \in P_{n}=\prod_{k=0}^{n-1}\{0,1, \ldots, N(k)\}$, we have

$$
\left|h\left(\alpha_{s}\right)-\sum_{k=0}^{N(n)} h\left(\alpha_{s k}\right)\right|<\varepsilon_{s}
$$

Then we obtain, for every $s \in P_{|s|}$,

$$
\begin{aligned}
h\left(\alpha_{s}\right)<\varepsilon_{s}+\sum_{n=0}^{N(|s|)} h\left(\alpha_{s n}\right)= & \varepsilon_{s}+\sum_{s u \in P_{|s|+1}} h\left(\alpha_{s u}\right) \\
& <\sum_{|t| \leqq 1} \varepsilon_{s t}+\sum_{s u \in P_{|s|+2}} h\left(\alpha_{s u}\right)<\cdots \\
& <\sum_{|t| \leqq n-1} \varepsilon_{s t}+\sum_{s u \in P_{|s|+n}} h\left(\alpha_{s u}\right),
\end{aligned}
$$

and, since $h\left(\alpha_{s}\right)=\sum_{|t|=h} h\left(\alpha_{s t}\right)$, we have

$$
\left|h\left(\alpha_{s}\right)-\sum_{s u \in P_{|s|+n}} h\left(\alpha_{s u}\right)\right|<\sum_{|t| \leq n-1} \varepsilon_{s t}
$$

Let $K=\prod_{n=0}^{\infty}\{0,1, \ldots, N(n)\} \subset \mathcal{N}$. It is clear that $K$ is compact. We are going to prove that the image $K^{\prime}$ of $K$ under the homeomorphism $T, K^{\prime}=T(K) \subset L$, has positive measure $\mu^{h}(T(K))$.

Let $K^{\prime} \subset \cup G_{n}$ be an open cover of $K^{\prime}$. There exists a $\rho>0$ so that every subset of $K^{\prime}$ of diameter $\leqq \rho$ is contained in the same $G_{n}$. As $\operatorname{diam}\left(B_{s} \cap K^{\prime}\right) \leqq \operatorname{diam}\left(B_{s} \cap L\right) \leqq \alpha_{s}+\delta_{s}$ and $\delta_{s k}<\alpha_{s k}<\operatorname{diam}\left(M_{J(s k)}\right)<\delta_{s} / 6$ we have

$$
\alpha_{s k}+\delta_{s k} \leqq \delta_{s} / 6+\delta_{s} / 6=\delta_{s} / 3<r_{s} / 2<(1 / 2)^{|s|+1}
$$

and $\operatorname{diam}\left(B_{s} \cap K^{\prime}\right)$ converges uniformly to zero as $|s| \rightarrow \infty$. There exists $n$ such that $|s|=n$ implies that $B_{s} \cap K^{\prime} \subset G_{m(s)}$ for some index $m(s)$. Let $J=\left\{m(s): s \in P_{n}\right\}$. It is clear that $K^{\prime} \subset \bigcup_{m \in J} G_{m}$ and $J$ is a finite set.

For every $m \in J$ there is a finite sequence $t$ such that $K^{\prime} \cap G_{m} \subset B_{t}$ and there are points $x, y \in K^{\prime} \cap G_{m}$ such that $x \in B_{t m_{1}}, y \in B_{t m_{2}}$ and $m_{1} \neq m_{2}$. It follows that $\operatorname{diam}\left(K^{\prime} \cap G_{m}\right) \geqq$ $\alpha_{t}-\delta_{l}$. For every $m \in J$, let $t_{m}$ denote the finite sequence $t$ determined in this way.

We have

$$
\sum_{m} h\left(\operatorname{diam}\left(G_{m}\right)\right) \geqq \sum_{m \in J} h\left(\operatorname{diam}\left(G_{m}\right)\right) \geqq \sum_{m \in J} h\left(\alpha_{t_{m}}-\delta_{t_{m}}\right) \geqq-\varepsilon+\sum_{m \in J} h\left(\alpha_{t_{m}}\right) .
$$

On the other hand

$$
h\left(\alpha_{t_{m}}\right)>\sum_{t_{m} \in P_{l^{\prime}, \mid+k}} h\left(\alpha_{t_{m} u^{u}}\right)-\sum_{|v| \leqq k-1} \varepsilon_{t_{m} v}
$$


so, if $j=\sup \left|t_{m}\right|$,

$$
\sum_{m} h\left(\operatorname{diam}\left(G_{m}\right)\right) \geqq-2 \varepsilon+\sum_{s \in P_{j}} h\left(\alpha_{s}\right) \geqq-3 \varepsilon+h\left(\alpha_{\theta}\right) \geqq h\left(\alpha_{0}\right) / 2>0 .
$$

This proves that $\mu^{h}\left(K^{\prime}\right) \geqq h\left(\alpha_{0}\right) / 2>0$.

Corollary 8. Lex $X$ be a complete metric vector space. $X$ has a small neighborhood of 0 if, and only if, $X$ is finite dimensional.

Proof. If $X$ is not locally compact, the construction of the theorem and Proposition 6 proves that every small subset of $X$ has an empty interior.

Otherwise, if $X$ is locally compact, it is finite dimensional and trivially small.

From Corollary 8 it follows that there exist separable metric spaces that are not small. A metric space $(X, d)$ is separable if, and only if, there exists an equivalent metric $d^{\prime}$ such that $\left(X, d^{\prime}\right)$ is totally bounded. So the concept of small metric space is not invariant under homeomorphism.

Christensen [1] defines the concept of Haar zero set in a abelian Polish group $G$ as follows: A universally measurable subset is a Haar zero set if, and only if, there exists a probability measure $\mu$ on $G$ such that every translation of the set is a zero set for the measure $\mu$. Here a set is universally measurable if it is measurable for every Radon probability defined on $G$.

Now if $A$ is small in $G$ and $G$ is not locally compact, there exists a continuous $h$ such that $\mu^{h}(A)=0$, and, by Theorem 7, a subset $L \subset G$ such that $0<\mu^{h}(L)<+\infty$. If we define the probability $\mu(X)=\mu^{h}(L \cap X) / \mu^{h}(L)$, we have that, since $\mu^{h}$ is an invariant measure, $\mu(a+A)=0$ for every $a \in G$.

So we have proved the following:

Proposition 9. Let $G$ be an abelian Polish group that is not locally compact. If $A$ is a universally measurable and small, then $A$ is a Haar zero set.

Let $X$ be an infinite dimensional separable Banach space. Let $Y$ be an hyperplane and $v \notin Y$. Let $\mu$ be a Lebesgue measure on the segment $[0, v]$. It is clear that $(a+Y) \cap[0, v]$ has at the most one point. Hence, if $Y$ is universally measurable, $Y$ is a Haar zero set. If $Y$ is closed, it is a Haar zero set but it is not small.

Theorem 10. Let $X$ be an infinite dimensional Banach space. The hyperplanes of $X$ are not small subsets.

Proof. Suppose that $Y$ is a small hyperplane. We shall construct a small Banach space $L$ of infinite dimension. We can assume that $Y$ is dense because, if $Y$ is closed, we can take $L=Y$.

Let $\left(z_{n}\right)$ be a normalized basic sequence contained in the hyperplane $Y$ and let $f$ be a linear form defined on $X$ such that $\operatorname{Ker}(f)=Y$.

Let $Z_{1}$ be the Banach space generated by the basic sequence $\left(z_{2 n}\right)$ and $Z_{2}$ that generated by $\left(z_{2 n+1}\right)$. Since $Z_{1}$ and $Z_{2}$ are infinite dimensional Banach spaces and $Y$ is small, there exists $u_{1} \in Z_{1}$ and $u_{2} \in Z_{2}$ such that $f\left(u_{1}\right) \neq 0$ and $f\left(u_{2}\right) \neq 0$. Substituting, if 
necessary, the base $\left(z_{k}\right)$ by $\left(\varepsilon_{k} z_{k}\right)$, where $\left|\varepsilon_{k}\right|=1$, we can assume that $f\left(u_{1}\right)>0>f\left(u_{2}\right)$,

$$
u_{1}=\sum u_{2 n}^{1} z_{2 n}, \quad u_{2}=\sum u_{2 n+1}^{2} z_{2 n+1} \quad \text { and } \quad u_{2 n}^{1} \geqq 0, \quad u_{2 n+1}^{2} \geqq 0 .
$$

Let $Z$ be the space generated by the base $\left(z_{n}\right)$. Let us renorm $Z$ so that

$$
\left\|\sum_{n}^{\infty} a_{k} z_{k}\right\| \leqq\left\|\sum_{1}^{\infty} a_{k} z_{k}\right\| .
$$

We define $Q_{n}: Z \rightarrow Z$ which transforms $\sum_{1}^{\infty} a_{k} z_{k}$ into $\sum_{k=n+1}^{\infty} a_{k} z_{k}$ and, for every $u \in Z$, set

$$
\|u\|\left\|=\sup _{n \geqq 0}\right\| Q_{n}(u) \|
$$

It is clear that, for every $z \in Z$, we have

$$
\begin{aligned}
& 0 \leqq\|u\|||<+\infty, \\
& \||\alpha u\|=|\alpha|\|| u\|, \\
& \|u+v\|\|\leqq\| u\|\mid\|+\|v\|, \\
& \|u\| \leqq\|u\| .
\end{aligned}
$$

Therefore $\|\mid\| \cdot \|$ is a norm in $Z$. By a standard argument, $Z$ with the new norm $\|\cdot \mid\|$, is complete. Finally

$$
\left\|Q_{m}(u)\right\|=\sup _{n}\left\|Q_{n}\left(Q_{m}(u)\right)\right\|=\sup _{n \geqq m}\left\|Q_{n}(u)\right\| \leqq\|u\| \|
$$

as we wished to prove.

In order to construct the small Banach space $L$, we define first the following set:

$$
B=\left\{\left(a_{k}\right): a_{k} \geqq 0, \sum a_{k} z_{k} \in Y \cap Z \text { and }\left\|\sum a_{k} z_{k}\right\|<1\right\} .
$$

The set $B$ satisfies the following assertions:

(1) $B$ is convex.

(2) Let $\left(a_{k}^{n}\right) \in B$ for every $n \in \mathbb{N}$ and let $r_{n} \geqq 0$ such that $\sum r_{n}=1$. Then there exists $\left(s_{k}\right) \in B$ such that $\sum_{n=1}^{\infty} r_{n} a_{k}^{n} \leqq s_{k}$ for every $k \in \mathbb{N}$.

Indeed, let $a_{n}=\sum_{k=1}^{\infty} a_{k}^{n} z_{k} \in U$ (where $U=\{z \in Z:\|z \mid\|<1\}$ denotes the unit ball of $Z$ ). Then $a=\sum r_{n} a_{n} \in U$. So, if $\left(z_{k}^{*}\right)$ is the sequence of coordinate functionals, we have

$$
z_{k}^{*}(a)=\sum_{n=1}^{\infty} r_{n} z_{k}^{*}\left(a_{n}\right)=\sum_{n=1}^{\infty} r_{n} a_{k}^{n} \geqq 0
$$

If $f(a)=0$, we can choose $s_{k}=\sum_{n=1}^{\infty} r_{n} a_{k}^{n}$. If $f(a) \neq 0$ we notice that $f\left(Q_{m}(z)\right)=f(z)$ for every $z \in Z$ and that there exists $\lambda>0$ such that either $f\left(a+\lambda u_{1}\right)=0$ or 
$f\left(a+\lambda u_{2}\right)=0$. Hence we substitute $a$ by $b=a+\lambda Q_{m}\left(u_{1}\right)$ or $b=a+\lambda Q_{m}\left(u_{2}\right)$. We take $m$ such that $\|b\| \mid<1$. Finally we choose $s_{k}$ such that $b=\sum s_{k} z_{k}$.

(3) $B \supset\left\{\left(a_{n}\right): a_{n} \geqq 0, \sum a_{n}<1\right.$ and there exists $N$ such that $n \geqq N$ implies $\left.a_{n}=0\right\}$. Since $\left\|\mid z_{n}\right\|\left\|=\sup _{m}\right\| Q_{m}\left(z_{n}\right)\left\|=\sup \left\{\left\|z_{n}\right\|, 0\right\}=\right\| z_{n} \|=1$ and $f\left(z_{n}\right)=0$, the property follows.

(4) There exists a sequence $\left(N_{k}\right)$ of real positive numbers such that $\lim N_{k}=+\infty$ and, if $\left(a_{k}\right) \in B$ and $m \in \mathbb{N}$, there exists $n \geqq m$ with $N_{n}\left(0, \ldots, 0, a_{n}, a_{n+1}, \ldots\right) \in B$.

Since $Z \cap Y$ is small, there exists a sequence of balls $B\left(v_{k}, r_{k}\right)$ such that $\lim r_{k}=0$ and $\bigcup_{k \geqq N} B\left(v_{k}, r_{k}\right) \supset U \cap Y$.

The vector space generated by $\left\{z_{1}, z_{2}, \ldots\right\}$ is dense in $Z$. Therefore we can assume that every $v_{k}$ belongs to this space. We can assume too that $v_{k}$ belongs to the space generated by $\left\{z_{1}, z_{2}, \ldots, z_{k}\right\}$ adding balls, if necessary.

Now let $\left(a_{k}\right) \in B$. As $a=\sum a_{k} z_{k} \in U \cap Y$, for every $m$, there exists $n \geqq m$ such that $a \in B\left(v_{n}, r_{n}\right)$. So $\left\|a-v_{n}\right\| \mid<r_{n}$. Therefore $\left\|Q_{n}\left(a-v_{n}\right)\right\| \mid<r_{n} ;$ but $Q_{n}\left(v_{n}\right)=0$, then \|\|$Q_{n}\left(a_{n}\right) \|<r_{n}$, that is $Q_{n}\left(a / r_{n}\right) \in U$, so $\sum_{k=n+1}^{\infty}\left(a_{k} / r_{n}\right) z_{k} \in U \cap Y$ and, consequently, $\left(1 / r_{n}\right)\left(0, \ldots, 0, a_{n+1}, a_{n+2}, \ldots\right) \in B$.

Now if we take $N_{n+1}=1 / r_{n}$, it is clear that $\lim N_{n}=+\infty$ and assertion (4) is proved.

With this properties of $B$, we can define $L$. Let $L$ be the set of sequences of real numbers $\left(x_{n}\right)$ such that there exist $\left(a_{n}\right) \in B$ and a positive number $M$ verifying $\left|x_{n}\right| \leqq M a_{n}$ for every $n \in \mathbb{N}$. Since $B$ is convex, it follows that $L$ is a vector space.

For every $\left(x_{n}\right) \in L$, we put $\left\|\left(x_{n}\right)\right\|=\inf \left\{r>0\right.$ : there exists $\left(a_{n}\right) \in B$ such that $\left|x_{n}\right| \leqq r a_{n}$ for every $n \in \mathbb{N}\}$. It is clear that $0 \leqq\left\|\left(x_{n}\right)\right\|<+\infty,\left\|\alpha\left(x_{n}\right)\right\|=|\alpha|\left\|\left(x_{n}\right)\right\|$ for every $\left(x_{n}\right) \in L$ and $\alpha \in \mathbb{R}$. From $\left|x_{n}\right| \leqq r a_{n}$ and $\left|y_{n}\right| \leqq s b_{n}$, it follows that

$$
\left|x_{n}+y_{n}\right| \leqq(r+s)\left(\frac{r}{r+s} a_{n}+\frac{s}{r+s} b_{n}\right)
$$

and we obtain $\left\|\left(x_{n}\right)+\left(y_{n}\right)\right\| \leqq\left\|\left(x_{n}\right)\right\|+\left\|\left(y_{n}\right)\right\|$.

For every natural number $k,\left\|z_{k}\right\|=1$. So, if $\left(a_{k}\right) \in B$, we have $a=\sum a_{k} z_{k} \in U$ and then

$$
a_{k}=\left|\left\|a_{k} z_{k}\right\|\right|=\left\|Q_{k-1}(a)-Q_{k}(a)\right\|\|\leqq\| Q_{k-1}(a)\left\|\left|+\left\|Q_{k}(a)\right\|\right| \leqq 2\right\||\|a\|| \leqq 2 .
$$

Let $\left(x_{n}\right) \in L$. If $\left|x_{n}\right| \leqq r a_{n}$, then $\left|x_{n}\right| \leqq r a_{n} \leqq 2 r$. So $\left|x_{n}\right| \leqq 2\left\|\left(x_{n}\right)\right\|$ and the proof that $\|\cdot\|$ is a norm on $L$ is finished.

We must prove now that $L$ is complete and small.

(a) Lis a Banach space

Consider the sequence $\left(w_{n}\right)$ in $L$ such that $\sum\left\|w_{n}\right\|<+\infty$. We have to prove that the series $\sum w_{n}$ is convergent in $L$.

Lwt $w_{n}=\left(x_{k}^{n}\right)$. We can suppose that $\sum_{1}^{\infty}\left\|w_{n}\right\|<1$. Let $r_{n}>\|w\|$ be such that $\sum r_{n}=1$. By definition of $\left\|w_{n}\right\|$, there exists $\left(a_{k}^{n}\right) \in B$ such that $\left|x_{k}^{n}\right| \leqq r_{n} a_{k}^{n}$ for every $k \in \mathbb{N}$. Then, for every $k \in \mathbb{N}$, the series $\sum_{n=1}^{\infty} x_{k}^{n}$ is convergent since $\left|x_{k}^{n}\right| \leqq 2 r_{n}$. Write $x_{k}=\sum_{n=1}^{\infty} x_{k}^{n}$ and 
consider the sequence $w=\left(x_{k}\right)$. We have $w \in L$ because

$$
\left|x_{k}\right| \leqq\left|\sum_{n=1}^{\infty} x_{k}^{n}\right| \leqq \sum_{n=1}^{\infty} r_{n} a_{k}^{n}=b_{k}
$$

and, by property (2) of $B$, there exists $\left(s_{k}\right) \in B$ such that $b_{k} \leqq s_{k}$ for every $k \in \mathbb{N}$.

Further, as

$$
\left|x_{k}-\sum_{n=1}^{N} x_{k}^{n}\right|=\left|\sum_{n=N+1}^{\infty} x_{k}^{n}\right| \leqq \sum_{n=N+1}^{\infty} r_{n} a_{k}^{n}=\left(\sum_{n=N+1}^{\infty} r_{n}\right)\left(\sum_{n=N+1}^{\infty} t_{n} a_{k}^{n}\right)
$$

where

$$
t_{n}=\left(\sum_{n=N+1}^{\infty} r_{n}\right)^{-1} r_{n} \text { satisfies } \sum_{n=N+1}^{\infty} t_{n}=1
$$

we obtain, by property (2) of $B$, that there exists $\left(s_{k}\right) \in B$ such that $\sum_{n=N+1}^{\infty} t_{n} a_{k}^{n} \leqq s_{k}$. So

$$
\left|x_{k}-\sum_{n=1}^{N} x_{k}^{n}\right| \leqq\left(\sum_{n=N+1}^{\infty} r_{n}\right) s_{k}
$$

This proves that $\left\|w-\sum_{n=1}^{N} w_{n}\right\| \leqq \sum_{n=N+1}^{\infty} r_{n}$ and concludes the proof of (a).

(b) $L$ is small.

Set $K_{n}=\left\{\left(x_{1}, \ldots, x_{n}, 0,0, \ldots\right):\left\|\left(x_{1}, \ldots, x_{n}, 0,0, \ldots\right)\right\| \leqq 1\right\} . K_{n}$ is contained in $L$ and it is obvious that it is compact. So we can choose points $w_{k}^{n} \in K_{n} k=1,2, \ldots, H_{n}$, such that

$$
K_{n} \subset \bigcup_{k=1}^{H_{n}} B\left(w_{k}^{n}, 1 / N_{n}\right)
$$

Now we arrange the balls $B\left(w_{k}^{n}, 2 / N_{n}\right)$ in a sequence $B\left(v_{k}, \rho_{k}\right)$. As $\lim N_{n}=+\infty$, it follows that $\lim \rho_{k}=0$.

The balls $B\left(v_{k}, \rho_{k}\right), k \geqq N$, cover $\left\{\left(x_{n}\right) \in L:\left\|\left(x_{n}\right)\right\|<1\right\}$ for every $N$. Indeed, let $w=\left(x_{n}\right) \in L$ be such that $\left\|\left(x_{n}\right)\right\|<1$. There exists a sequence $\left(a_{k}\right) \in B$ verifying $\left|x_{n}\right| \leqq a_{k}$.

By property (4), given $m \in \mathbb{N}$, there exists $n \geqq m$ such that $N_{n}\left(0, \ldots, a_{n}, a_{n+1}, \ldots\right) \in B$. It follows that $\left(0, \ldots, 0, x_{n}, x_{n+1}, \ldots\right)=v_{1} \in L$ and $\left\|v_{1}\right\| \leqq 1 / N_{n}$. Moreover $\left(x_{1}, \ldots, x_{n-1}, 0,0, \ldots\right)=$ $v_{2} \in L$ and $\left\|v_{2}\right\| \leqq\|w\|<1$. So $v_{2} \in K_{n}$ and, by the above construction, there exists $w_{k}^{n}$ such that $\left\|v_{2}-w_{k}^{n}\right\| \leqq 1 / N_{n}$.

Then we have $w=v_{1}+v_{2}$,

$$
\left\|w_{k}^{n}-w\right\| \leqq\left\|w_{k}^{n}-v_{2}\right\|+\left\|v_{1}\right\| \leqq 2 / N_{n}
$$

and $w \in B\left(w_{k}^{n}, 2 / N_{n}\right)$.

As $L$ is infinite dimensional, (a) and (b) contradict Corollary 8 so $Y$ can not be small. 
Theorem 11. Let $X$ be a normed space. The unit ball of $X$ can be covered by a sequence of balls whose radii $r_{n}<1$ converge to zero if, and only if, $X$ is small.

Proof. If $X$ is small, it is obvious that $B(0,1)$ can be covered by a sequence of balls $B\left(x_{n}, r_{n}\right)$ such that $r_{n}<1$ and $r_{n}$ converge to zero.

Assume that $B(0,1) \subset \bigcup_{n=0}^{\infty} B\left(x_{n}, r_{n}\right)$ with $r_{n}<1$ and $\lim r_{n}=0$. We will prove that $B(0,1)$, and hence $X$, is small.

If $B(0,1) \subset \bigcup_{n=0}^{\infty} B\left(x_{n}, r_{n}\right)$, it follows that, for every $r>0$ and $a \in X, B(a, r) \subset \bigcup_{n=0}^{\infty} B(a+$ $\left.r x_{n}, r r_{n}\right)$.

Let $s=\langle s(0), s(1), \ldots, s(n-1)\rangle$ be a finite sequence of natural numbers. We say that $|s|=n$ is the length of $s$. We define

$$
x_{s}=x_{s(0)}+r_{s(1)} x_{s(1)}+\cdots+r_{s(n-1)} x_{s(n-1)}
$$

and $r_{s}=r_{s(0)} r_{s(1)} \ldots r_{s(n-1)}$.

Now we consider the balls $B_{s}=B\left(x_{s}, r_{s}\right)$ with $|s| \geqq 1$. We have

$$
B_{s} \subset \bigcup_{n=0}^{\infty} B\left(x_{s}+r_{s} x_{n}, r_{s} r_{n}\right)=\bigcup_{n=0}^{\infty} B_{s n}
$$

Let $S_{1}$ be the set of finite sequences of natural numbers of length $\geqq 1$ and $F \subset S_{1}$ a finite subset. Then

$$
\bigcup_{s \in S_{1} \backslash F} B_{s}=\bigcup_{s \in S_{1}} B_{s} \supset B(0,1)
$$

Indeed, if $t \in F$ is of maximal length in $F$,

$$
B_{t}=\bigcup_{n=0}^{\infty} B_{t n} \subset \bigcup_{s \in S_{1} \backslash F} B_{s}
$$

It is clear that the set of balls $B_{s}$ is countable.

If we form a sequence with these balls $\left(B\left(z_{n}, \rho_{n}\right)\right)$, then $\lim \rho_{n}=0$. Indeed, given $\varepsilon>0$, if $r_{s}>\varepsilon$, we have $r_{s(0)} r_{s(1)} \ldots r_{s(n-1)}>\varepsilon$. Then $\left(\sup r_{k}\right){ }^{|s|}>\varepsilon$. But $\left(\sup r_{k}\right)<1$ and $\left(\sup r_{k}\right)^{|s|}>\varepsilon$ imply $|s| \leqq m$. As $r_{n}<1, r_{s(0)} r_{s(1)} \ldots r_{s(n-1)}>\varepsilon$ implies $r_{s(i)}>\varepsilon$ and therefore $s(i) \leqq k$. So $r_{s} \leqq \varepsilon$ except for a finite number of sequences $s \in S_{1}$. There are at most $(k+1)^{m}$ sequences such that $r_{s}>\varepsilon$.

Theorem 12. Let $X$ be a separable Banach space of infinite dimension. There exists a small subset $A \subset X$ such that $A+A=X$.

Proof. Let $\left(x_{n}\right)$ be a dense sequence in $X$. Let $\left(r_{n}\right)$ be a sequence of positive real numbers such that $\lim r_{n}=0$. Define

$$
A=\bigcap_{N} \bigcup_{n \in N} B\left(x_{n}, r_{n}\right)
$$


It is clear that $A$ is small. Also, as $\bigcup_{n \geqq N} B\left(x_{n}, r_{n}\right)$ is an open dense set, it follows that $B=X \backslash A$ is a set of first category.

Let $x \in X$. The set $(x-B) \cup B$ is of first category. Choose $y_{1} \notin(x-B) \cup B$ and define $y_{2}=x-y_{1}$. Then we have $x=y_{1}+y_{2}, \quad y_{1} \notin B$ and $y_{2}=x-y_{1} \notin x-(x-B)=B$. Hence $x \in A+A$.

Remark. This set $A$ is not contained in a countable union of totally bounded sets, $\cup P_{n}$. Indeed, if $A \subset \cup P_{n}$, then $A \subset \cup \bar{P}_{n}$. But $\bar{P}_{n}$ is compact, so closed and with empty interior. Then $X=A \cup B$ would be of first category.

\section{REFERENCES}

1. J. P. R. Christensen, Topology and Borel Structure (North-Holland, Amsterdam, 1974).

2. J. ConNetr, On covering the unit ball in normed spaces, Canad. Math. Bull. 14 (1971), 107109.

3. J. ConNert, On covering the unit ball in a Banach space, J. London Math. Soc. (2) 7 (1973), 291-294.

4. T. Dominguez Benavides, Some properties of the set and ball measures of non-compactness and applications, J. London Math. Soc. (2) 34 (1986), 120-128.

5. D. H. Fremlin, Consequences of Martin's Axiom (Cambridge University Press, Cambridge, 1984).

6. T. W. Korner, Some covering theorems for infinite dimensional vector spaces, J. London Math. Soc. (2) 2 (1970), 643-646.

7. K. Kuratowski, Sur les espaces completes, Fund. Math. 15 (1939), 301-309.

8. C. A. Rogers, Hausdorff Measures (Cambridge University Press, Cambridge, 1970).

Universidad de Sevilla

Facultad de Matemáticas

APDO. 1160

41080-SeVILLA

SPAIN 